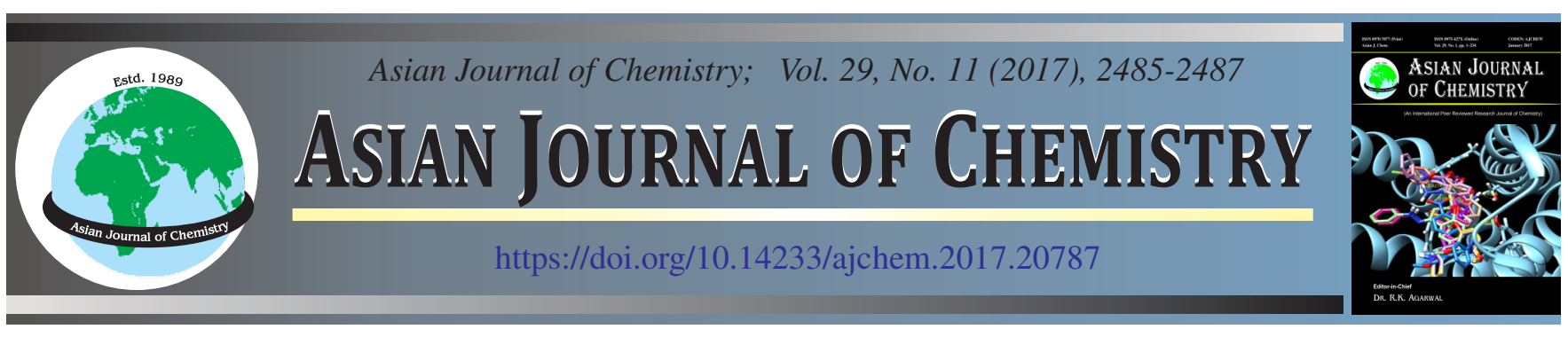

\title{
Regeneration of Carbonyl Compounds by Deoximation of Piperidine-4-one Oximes with Quinolinium Flurochromate in Presence of Montmorillonite K10 Clay
}

\author{
T. ViMALA ${ }^{*}$ and U.D. LiNGESWARI
}

PG and Research Department of Chemistry, Seethalakshmi Ramaswami College, Tirichirappalli-620 002, India

*Corresponding author: E-mail: vimalsrc@gmail.com

Received: 17 May 2017;

Accepted: 30 June 2017;

Published online: 29 September 2017;

AJC-18577

\begin{abstract}
The regeneration of oximes from 2,6-diphenyl piperidine-4-one oximes (PPO) and its alkyl substituted derivatives (3-alkyl PPO and 3,5dimethyl PPO) by quinolinium flurochromate under microwave irradiation in the presence of montmorillonite K10 clay leads to the formation of ketone in a good yield.
\end{abstract}

Keywords: Piperidine 4-one oximes, Quinolinium flurochromate, Microwave irradiation, Montmorillonite K10 clay.

\section{INTRODUCTION}

Oximes and semicarbazones of aldehydes and ketones are highly crystalline compounds. Deprotection of these derivatives is an efficient method for the isolation, purification and characterization of carbonyl compounds [1-3]. Oximes not only serve as the protecting groups for carbonyl compounds but also have other uses such as the preparation of nitrites, amides via Beckman rearrangement. In addition, oximes can be prepared from non-carbonyl compounds and therefore regeneration of carbonyl compounds from their oximes is an important reaction. This is especially true with natural products. Various methods are adopted for the deoximation process to obtain aldehydes and ketones [4,5]. Oxidation, reduction and hydrolysis are the common methods using organic and inorganic reagents. Besides some surface templates processes and photochemical processes are also used to give the required products [6]. The solvent free deoximation of protected carbonyl compounds by ammonium persulphate on silica [7] and regeneration of carbonyl compounds from aromatic semicarbazones by ammonium persulphate impregnated on montmorillonite K 10 clay in microwave or ultra sound irradiation [8] was also reported. Poly[N-bromobenzene-1,3-disulphonamide] [9] is used for the conversion of oximes and semicarbazones in solid state to their corresponding carbonyl compounds [10,11].

However, most of the procedures used for this type of reaction require a use of solid support or other axillaries, solvents for the extraction from the solid supports, high temperature, long reaction time, expensive and not readily available reagents and tedious work-up procedures. Consequently, there is a demand for the development of this process using readily available reagents which operate under extremely mild reaction conditions. Microwave reaction is a green method for efficient deprotection [12,13]. Recent developments in microwave accelerated and solvent free approach involves the exposure of neat reactants to microwave irradiation in conjugation with the use of supported reagents as catalyst give good yields. Quinolinium flurochromate (QFC), in the Cr(IV) oxidant series is one of the efficient and interesting oxidizing agent [14-17]. Quinolinium flurochromate is found to have several advantages over similar oxidizing agents due to its special characteristics such as less acidity, better solubility in non-aqueous solvents, shorter reaction times and forming products in high yield. It behaves as two electron oxidant in majority of the reactions so far studied. A perusal of literature revels that no work has been reported on the deprotection of 3-alkyl-2,6-diphenyl piperidin-4-one oximes with QFC by microwave method.

\section{EXPERIMENTAL}

Quinoline was distilled at its boiling point. Chromium trioxide, hydrofluoric acid, hydroxylamine hydrochlorite, silica gel, dichloro methane, diethyl ether, petroleum ether, montmorillonite clay K10 and ethyl acetate were used as such (Analar grade).

Quinolinium flurochromate (QFC), a complex of chromic acid, quinoline and hydrogen fluoride was prepared as reported earlier [14]. Chromium(VI) oxide (15 g:0.15 mol) was dissolved in water $(25 \mathrm{~mL})$ in a polythene beaker and $40 \%$ hydrofluoric acid $(11.3 \mathrm{~mL}: 0.23 \mathrm{~mol})$ added to it with stirring at 
room temperature. To this clear solution, distilled quinoline (17.7 mL:0.15 mol) was added slowly with stirring. The mixture was heated on a steam-bath for $0.5 \mathrm{~h}$, then cooled to room temperature and allowed to stand for $1 \mathrm{~h}$. The bright red-orange crystalline quinolinium flurochromate was isolated by filtration and dried in vacuo for $1 \mathrm{~h}$ (m.p. $163{ }^{\circ} \mathrm{C}$ and $162-163{ }^{\circ} \mathrm{C}$ ). All the oximes were prepared from the respective ketones by the reported procedure $[18,19]$.

Microwave irradiation process: Microwave chemistry is the science of applying microwave radiation to chemical reactions. Microwave acts as high frequency electric fields and generally heat any material containing mobile electric charges, such as polar molecules in a solvent or conducting ions in a solid. Montmorillonite clay K10 known as a Brønsted acid have a great impact on organic synthesis. These solid supports with lamellar swelling structure and large surface area enhance selectivity in synthetic organic chemistry. Avoding organic solvents during the reaction in organic synthesis lead to a clean, efficient and economical technology. In solid state reaction, work-up is considerably simplified, cost is reduced and increased amounts of reactants can be used in the same equipment [20].

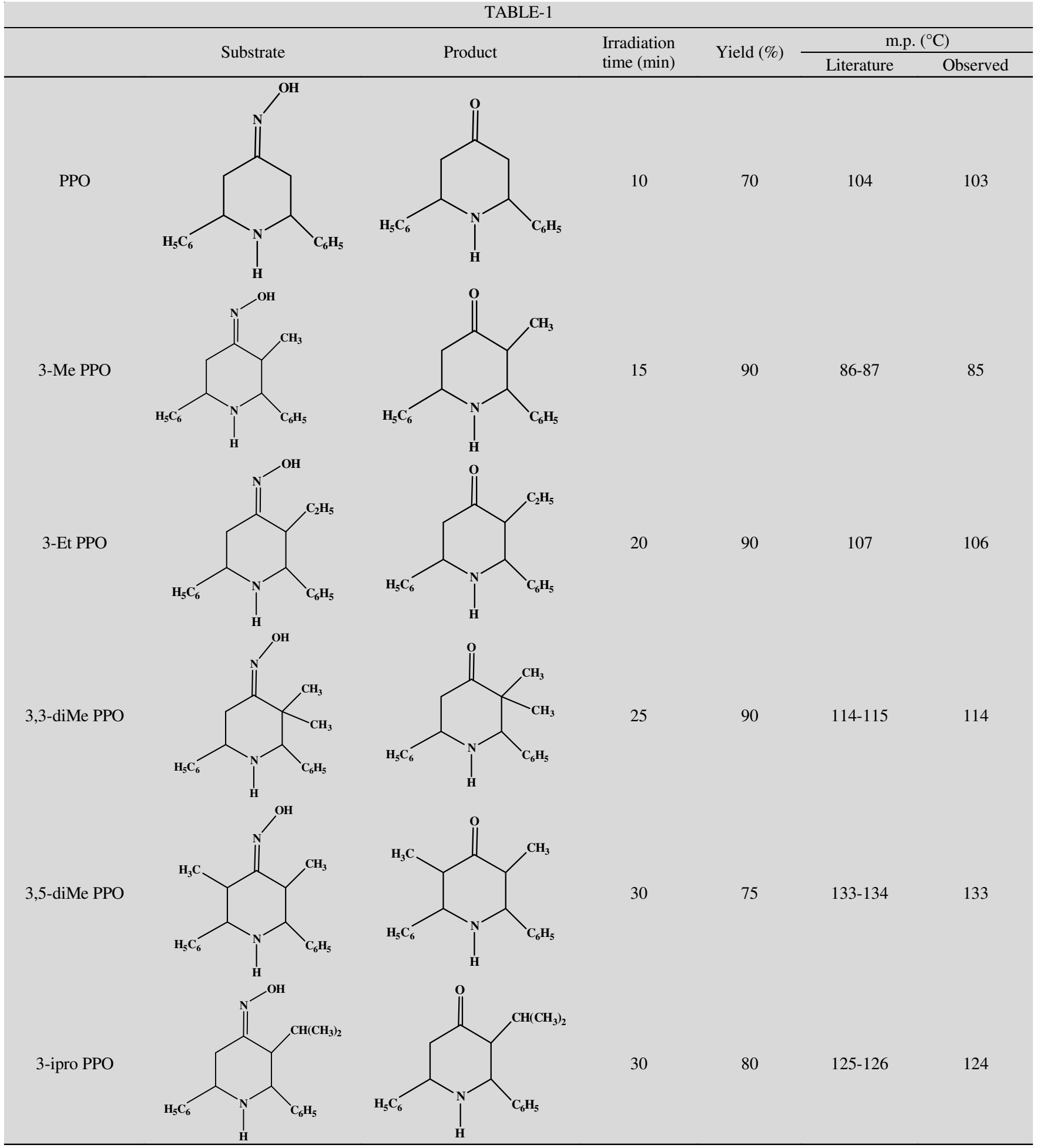


3-Alkyl-2,6-diphenyl piperidin-4-one oxime was mixed with quinolinium flurochromate $(4 \mathrm{mmol})$ with $2 \mathrm{~g}$ of montmorillonite clay K10 and irradiated in microwave oven (LG. model No. MH2043DW) for an appropriate time. The progress of the reaction was monitored by thin layer chromatography. After completion of the reaction the products were recovered by passing the reaction mixture through column chromatography of silica. (200-300 mesh, eluted with mixture of petroleum ether and ethyl acetate).

Separation of product by column chromatography: A column of $5 \times 1 \mathrm{~cm}$ was packed with silica gel by making slurry with dichloromethane. It was dried in an oven and used for eluting the products. The product dissolved in dichloromethane and diethyl ether (9:1). After repeated eluting and washing the fractions obtained were collected together and evaporated to get the solid ketone.

Thin layer chromatography: TLC plate is prepared by dipping it in slurry of silica gel in benzene. Petroleum ether ethyl acetate mixture (9:1) is used as an eluent. It is observed that the $\mathrm{R}_{\mathrm{f}}$ values of oximes and corresponding ketones formed in the oxidation process are found to be different while that of pure ketone and the product ketone are found to be the same indicating the formation of ketone.

\section{RESULTS AND DISCUSSION}

The product was identified by determining their melting point (Table-1). A qualitative test was also carried out to confirm the formation of ketone.

\section{FTIR spectral analysis}

3-Alkyl-2,6-diphenyl piperidin-4-one oxime: In the FTIR spectra of 3-methyl-2,6-diphenyl piperidin-4-one oxime (3-MePPO) and 3-ethyl-2,6-diphenyl piperidin-4-one oxime (3-EtPPO) the intense peaks at 1573.10 and $1576.34 \mathrm{~cm}^{-1}$ are due to the presence of $\mathrm{C}=\mathrm{N}$ stretching vibration, respectively. The stretching vibration at 758.70 and $699.32 \mathrm{~cm}^{-1}$ and 785.65 and $701.7 \mathrm{~cm}^{-1}$ shows the presence of phenyl ring. The peaks at 1484.65 and $1489.68 \mathrm{~cm}^{-1}$ are due to $\mathrm{C}-\mathrm{H}$ bending vibrations of alkyl groups in 3-MePPO and 3-EtPPO skeleton, respectively. The OH group of 3-MePPO and 3-EtPPOO exhibit vibrations at 3396.7 and $3273.24 \mathrm{~cm}^{-1}$. In the FTIR spectra of 3isopropyl-2,6-diphenyl piperidin-4-one oxime (3-iprPPO) the intense peaks at $1598 \mathrm{~cm}^{-1}$ is due to the presence of $\mathrm{C}=\mathrm{N}$ stretching vibration. The stretching vibration at $756.45 \mathrm{~cm}^{-1}$ and $679.95 \mathrm{~cm}^{-1}$ shows the presence of phenyl ring. The peak at $1447 \mathrm{~cm}^{-1}$ is due to the $\mathrm{C}-\mathrm{H}$ bending vibrations of isopropyl group. The sharp peaks at $1700.45,1705.69$ and $1701.09 \mathrm{~cm}^{-1}$ are due to the carbonyl groups present in corresponding ketones. Formation of ketone as a product is further confirmed by the blue shift in $\lambda_{\max }$ value of UV-visible spectrum of the product ketones compared to oximes [21,22].

\section{ACKNOWLEDGEMENTS}

The authors are gratefully acknowledge the financial support provided by the University Grants Commission, New Delhi, India to carry out this work.

\section{REFERENCES}

1. W. Carruthers, Some Modern Methods of Organic Synthesis, Cambridge University Press, Cambridge, London, p. 345 (1986).

2. K.L. Williamson, Macroscale and Microscale Organic Experiments, Houghton-Miffin, Baston, edn 3, p. 426 (1999).

3. T.W. Greene and P.G. Wust Protective Groups in Organic Synthesis, John Wily \& Sons, New York, edn 3 (1999).

4. A.P. Sikar and P.J. Das, Indian J. Chem., 46B, 1208 (2007).

5. S. Sahu, S. Sahu, S. Petal, S. Dash and B.K. Mishra, Indian J.Chem., 47B, 259 (2008).

6. C. Saravanselvi, N. Somasundaram, S. Vijaikumar and C. Srinivasan, Photochem. Photobiol. Sci., 1, 607 (2002); https://doi.org/10.1039/B203721C.

7. R.S. Varma and H.M. Meshram, Tetrahedron Lett., 38, 5427 (1997); https://doi.org/10.1016/S0040-4039(97)01213-6.

8. $\quad$ R.S. Varma and H.M. Meshram, Tetrahedron Lett., 38, 7973 (1997); https://doi.org/10.1016/S0040-4039(97)10143-5.

9. H. Veisi, R. Ghorbani-Vaghei and M.A. Zolfigol, Org. Prep. Proc. Int., 43, 489 (2011); https://doi.org/10.1080/00304948.2011.629553.

10. R. Ghorbani-Vaghei and E. Shahloazee, J. Braz. Chem. Soc., 16, 647 (2005).

11. P.T. Perumal, M. Anniyappan and D. Muralidharan, J. Chem. Sci., 116, 261 (2004); https://doi.org/10.1007/BF02708276.

12. K. Banerjee, A.K. Mitra and A. Patra, Indian J. Chem., 45 B, 537 (2006).

13. Y.-F. Zohu, F. Lin, X. L. Lu, C. Zhanghe, Q. Wang, X.-N. Zou and J.-D. Lou, Oxid. Commun., 35, 45 (2012).

14. V. Murugesan and A. Pandurangan, Indian J. Chem., 31B, 377 (1992).

15. D.S. Bose and A.V. Narasaiah, Synth. Commun., 30, 1153 (2000); https://doi.org/10.1080/00397910008087132.

16. G.A. Rajkumar and V. Sivamurugan, Indian J. Chem., 43B, 936 (2004).

17. M. Tajbakhsh, K. Alimohammadi, H. Behmadi and M. Barghamadi, J. Chem. Res., 2005, 35 (2005); https://doi.org/10.3184/0308234053431194.

18. V. Baliah, K. Pandiyarajan and M.A. Lakshmanan, Indian J. Chem., 16B, 72 (1978)

19. C.R. Noller and V. Baliah, J. Am. Chem. Soc., 70, 3853 (1948); https://doi.org/10.1021/ja01191a092.

20. X. Jing, D. Yuan and L. Yua, Adv. Synth. Catal., 359, 1194 (2017); https://doi.org/10.1002/adsc.201601353.

21. J.R. Dyer, Applications of Absorption Spectroscopy of Organic Compounds, Prentice Hall of India Pvt. Ltd., New Delhi, India (1991).

22. P.S. Kalsi, Spectroscopy of Organic Compounds, John Wiley \& Sons (Asia) Pvt. Ltd. (1995). 